Faculty of Mathematical Sciences

\section{University of Twente}

University for Technical and Social Sciences
P.O. Box 217

7500 AE Enschede

The Netherlands

Phone: +31-53-4893400

Fax: +31-53-4893114

Email: memo@math.utwente.nl

\author{
Memorandum No. 1554 \\ On the convergence to stationarity \\ of birth-death processes \\ P. Coolen-Schrijner ${ }^{1}$ And E.A. van Doorn
}

November 2000

ISSN 0169-2690

\footnotetext{
${ }^{1}$ Dept. of Mathematical Sciences, University of Durham, Science Laboratories, South Road, Durham DH1 3LE, United Kingdom
} 


\title{
On the convergence to stationarity of birth-death processes
}

\author{
Pauline Coolen-Schrijner* and Erik A. van Doorn**
}

*Department of Mathematical Sciences

University of Durham

Science Laboratories, South Road, Durham DH1 3LE, UK

E-mail: pauline.schrijner@durham.ac.uk

${ }^{* *}$ Faculty of Mathematical Sciences

University of Twente

P.O. Box 217, 7500 AE Enschede, The Netherlands

E-mail: doorn@math.utwente.nl

November 14, 2000

\begin{abstract}
Taking up a recent proposal by Stadje and Parthasarathy in the setting of the many-server Poisson queue, we consider the integral $\int_{0}^{\infty}\left[\lim _{u \rightarrow \infty} E(X(u))-E(X(t))\right] d t$ as a measure of the speed of convergence towards stationarity of the process $\{X(t), t \geq 0\}$, and evaluate the integral explicitly in terms of the parameters of the process in the case that $\{X(t), t \geq 0\}$ is an ergodic birth-death process on $\{0,1, \ldots\}$ starting in 0 . We also discuss the discrete-time counterpart of this result, and examine some specific examples.
\end{abstract}

Keywords and phrases: birth-death process, random walk, speed of convergence 2000 Mathematics Subject Classification: 60J80 


\section{Introduction}

Let $X(t)$ be the number of customers at time $t$ in a stable $M / M / c$ queueing system and suppose that the system is initially empty. The process $\{X(t), t \geq$ $0\}$ is then stochastically increasing, and, as a consequence, $E(X(t))$ converges monotonically to its limiting value

$$
M \equiv \lim _{t \rightarrow \infty} E(X(t)) .
$$

This has recently motivated Stadje and Parthasarathy [10] to propose the quantity

$$
\int_{0}^{\infty}[M-E(X(t))] d t
$$

as a measure of the speed of convergence as $t \rightarrow \infty$ of the distribution of $X(t)$ to the stationary distribution of the number of customers in an $M / M / c$ system. They subsequently evaluate the integral (1) explicitly in terms of the number of servers $c$, and the arrival and service rates of the system.

Clearly, the process $\{X(t), t \geq 0\}$ constitutes a birth-death process. Moreover, any birth-death process on the nonnegative integers which starts in state 0 is stochastically increasing (see, for example, Kijima [9, Section 4.8]). It is therefore natural to ask whether the result of Stadje and Parthasarathy can be extended into the more general setting of birth-death processes. The purpose of this paper is to resolve this question in the affirmative. So in what follows $\mathcal{X} \equiv\{X(t), t \geq 0\}$ will be an ergodic birth-death process taking values in $\mathcal{N} \equiv\{0,1, \ldots\}$ with birth rates $\left\{\lambda_{j}, j \in \mathcal{N}\right\}$ and death rates $\left\{\mu_{j}, j \in \mathcal{N}\right\}$, all strictly positive except $\mu_{0}=0$. Throughout we will assume $X(0)=0$ and use the notation

$$
p_{j}(t) \equiv \operatorname{Pr}\{X(t)=j \mid X(0)=0\}, \quad j \in \mathcal{N}, t \geq 0,
$$

and

$$
p_{j} \equiv \lim _{t \rightarrow \infty} p_{j}(t), \quad j \in \mathcal{N} .
$$

The speed of convergence to stationarity of the process $\mathcal{X}$ is usually characterized by the decay parameter

$$
\gamma(\mathcal{X}) \equiv \sup \left\{\gamma \geq 0 \mid p_{j}-p_{j}(t)=\mathcal{O}(\exp (-\gamma t)) \text { as } t \rightarrow \infty\right\}
$$


(which is independent of $j$ ), or its reciprocal $r(\mathcal{X}) \equiv 1 / \gamma(\mathcal{X})$, the relaxation time (see, for example, [1] and [12]). If $M \equiv \lim _{t \rightarrow \infty} E(X(t))<\infty$ we also have

$$
r(\mathcal{X})=\inf \{r>0 \mid M-E(X(t))=\mathcal{O}(\exp (-t / r)) \text { as } t \rightarrow \infty\},
$$

the infimum of an empty set being infinity. The relaxation times of many specific birth-death processes are known, but there exists no general expression for $r(\mathcal{X})$ in terms of the birth and death rates of $\mathcal{X}$. Since, as we will show, the integral (1) can be evaluated explicitly in terms of the birth and death rates of $\mathcal{X}$ it may be an attractive alternative to $r(\mathcal{X})$ as a one-parameter characterization of the speed of convergence. Rather than (1), however, we propose its normalized value

$$
m(\mathcal{X}) \equiv \int_{0}^{\infty}[1-E(X(t)) / M] d t
$$

as an alternative to $r(\mathcal{X})$ as a measure of the speed of convergence towards stationarity of the process $\mathcal{X}$.

The rest of the paper is organised as follows. After presenting some preliminary results on birth-death processes in Section 2, we will obtain our main result - an explicit expression for the integral (1) in terms of the birth and death rates - in Section 3. The expression will be evaluated for some specific birth-death processes in Section 4. In particular, we will compare our findings with those of Stadje and Parthasarathy [10] (and find a discrepancy). Finally, in Section 5 , we consider birth-death processes in discrete time, and show that a similar result may be obtained in this setting by performing a suitable transformation, provided the birth and death probabilities satisfy certain requirements.

\section{Preliminaries}

The potential coefficients of the birth-death process $\mathcal{X} \equiv\{X(t), t \geq 0\}$ are defined by

$$
\pi_{0} \equiv 1 \text { and } \pi_{j} \equiv \frac{\lambda_{0} \lambda_{1} \ldots \lambda_{j-1}}{\mu_{1} \mu_{2} \ldots \mu_{j}}, \quad j \geq 1 .
$$

Since $\mathcal{X}$ is assumed to be ergodic these constants must satisfy the condition

$$
K \equiv \sum_{j=0}^{\infty} \pi_{j}<\infty .
$$


We will additionally assume

$$
\sum_{j=0}^{\infty}\left(\lambda_{j} \pi_{j}\right)^{-1}=\infty
$$

ensuring that $\mathcal{X}$ is uniquely determined by its birth and death rates (see [4]).

It is well known that

$$
p_{j} \equiv \lim _{t \rightarrow \infty} p_{j}(t)=\frac{\pi_{j}}{K}, \quad j \in \mathcal{N},
$$

while (see, for example, Holewijn and Hordijk [3])

$$
\lim _{t \rightarrow \infty} E(X(t))=E(X),
$$

$X$ denoting a random variable with distribution $\left\{p_{j}, j \in \mathcal{N}\right\}$. Evidently, we will assume throughout that

$$
E(X)=\sum_{j=0}^{\infty} j p_{j}<\infty
$$

It will be convenient to introduce the quantities

$$
\tau_{j} \equiv p_{j} \sum_{k=0}^{j-1}\left(\lambda_{k} p_{k}\right)^{-1} \sum_{\ell=k+1}^{\infty} p_{\ell}, \quad j \geq 0,
$$

and

$$
T \equiv \sum_{j=0}^{\infty} \tau_{j}
$$

Here, and henceforth, the empty sum should be interpreted as zero (so that $\left.\tau_{0} \equiv 0\right)$. By interchanging summations it is easily seen that

$$
T=\sum_{k=0}^{\infty}\left(\lambda_{k} p_{k}\right)^{-1}\left(\sum_{\ell=k+1}^{\infty} p_{\ell}\right)^{2},
$$

which may be finite or infinite.

\section{The main result}

In this section we will first evaluate the integrals

$$
I_{j} \equiv \int_{0}^{\infty}\left[p_{j}(t)-p_{j}\right] d t, \quad j \geq 0,
$$


after which the value of the integral (1) will follow as a corollary. Since $p_{j}(t)$ is a unimodal function (see Keilson [8]) the integrals $I_{j}$ exist, but may be infinite.

The integrals $I_{j}$ have been evaluated explicitly by Whitt [14, Proposition $6]$ in the setting of a birth-death process with finite state space $\{0,1, \ldots, n\}$. Letting $n$ tend to infinity in the expression for $I_{j}$ given by Whitt yields after a little algebra

$$
I_{j} \equiv \int_{0}^{\infty}\left[p_{j}(t)-p_{j}\right] d t=T p_{j}-\tau_{j}, \quad j \geq 0,
$$

with the interpretation that $I_{j}=\infty$ whenever $T=\infty$. We have verified this result by substituting in (11) the spectral representation for $p_{j}(t)$ developed by Karlin and McGregor [4] and exploiting the technique suggested by Karlin and McGregor [5, p. 399] to evaluate the resulting integral.

We are now in a position to state our main result.

Theorem 1 If $\sum_{0}^{\infty} j \tau_{j}<\infty$, then

$$
\int_{0}^{\infty}[E(X)-E(X(t))] d t=\sum_{j=0}^{\infty} j \tau_{j}-T E(X),
$$

whereas the integral is infinite otherwise.

Proof. Since $\mathcal{X}$ is stochastically increasing, we have

$$
\sum_{j=0}^{k}\left(p_{j}(t)-p_{j}\right)>0, \quad k \geq 0 .
$$

We also observe

$$
E(X)-E(X(t))=\sum_{k=1}^{\infty} \sum_{j=k}^{\infty}\left(p_{j}-p_{j}(t)\right)=\sum_{k=1}^{\infty} \sum_{j=0}^{k-1}\left(p_{j}(t)-p_{j}\right) .
$$

It follows that

$$
E(X)-E(X(t))>p_{0}(t)-p_{0},
$$

and hence, by (12), the integral is infinite if $T=\infty$. Now assuming $T<\infty$, and using (15) and the fact that $\sum I_{j}=0$, we can write

$$
\int_{0}^{\infty}[E(X)-E(X(t))] d t=\sum_{k=1}^{\infty} \sum_{j=0}^{k-1} I_{j}=-\sum_{k=1}^{\infty} \sum_{j=k}^{\infty} I_{j}=-\sum_{j=1}^{\infty} j I_{j},
$$

the interchange of integration and summation being justified by (14). In view of (8) and (12) the theorem follows. 


\section{Examples}

To check the theorem we first look at a process for which the value of the integral (1) is available. Namely, we let $\mathcal{X} \equiv\{X(t), t \geq 0\}$ be the number of customers in the $M / M / \infty$ queue, which is a birth-death process with rates

$$
\lambda_{j}=\lambda \text { and } \mu_{j}=j \mu, \quad j \in \mathcal{N} \text {. }
$$

It is well known (see, for example, Feller [2, p. 461]) that when the system starts empty the mean number of customers in the system at time $t$ is given by

$$
E(X(t))=\frac{\lambda}{\mu}\left(1-e^{-\mu t}\right), \quad t \geq 0
$$

so that

$$
\int_{0}^{\infty}[E(X)-E(X(t))] d t=\frac{\lambda}{\mu^{2}} .
$$

This result can indeed be recovered - albeit somewhat tediously - by evaluating the right-hand side of (13). For completeness' sake we note that the convergence measures (2) and (3) for this process are given by

$$
m(\mathcal{X})=r(\mathcal{X})=\frac{1}{\mu} .
$$

Our second example is the birth-death process $\mathcal{X}$ with rates

$$
\lambda_{j}=\lambda /(j+1) \text { and } \mu_{j+1}=\mu, \quad j \geq 0,
$$

which may be interpreted as the process of the number of customers in a queueing system in which customers are discouraged by queue length (see, for example, [11]). In this case no simple expression for $E(X(t))$ is available. To evaluate the right-hand side of (13) we write

$$
a \equiv \lambda / \mu
$$

and note that

$$
K=e^{a} \text { and } E(X)=a .
$$

Moreover, letting

$$
f_{j}(a) \equiv \sum_{\ell=1}^{\infty} \frac{(j+1) !}{(j+\ell) !} a^{\ell}, \quad j \geq 0,
$$


we readily obtain

$$
\tau_{j}=\frac{1}{\lambda} e^{-a} \frac{a^{j}}{j !} \sum_{k=0}^{j-1} f_{k}(a), \quad j \geq 0,
$$

so that

$$
T=\frac{1}{\lambda} e^{-a} \sum_{j=1}^{\infty} \frac{a^{j}}{j !} \sum_{k=0}^{j-1} f_{k}(a) \text { and } \sum_{j=1}^{\infty} j \tau_{j}=\frac{a}{\lambda} e^{-a} \sum_{j=0}^{\infty} \frac{a^{j}}{j !} \sum_{k=0}^{j} f_{k}(a) .
$$

Substitution of these results in (13) gives us

$$
\int_{0}^{\infty}[E(X)-E(X(t))] d t=\frac{a}{\lambda} e^{-a} \sum_{j=0}^{\infty} \frac{a^{j}}{j !} f_{j}(a),
$$

which, after substitution of (18) and (19) and some algebra, reduces to

$$
\int_{0}^{\infty}[E(X)-E(X(t))] d t=\frac{\lambda(\lambda+2 \mu)}{2 \mu^{3}} .
$$

It now follows that

$$
m(\mathcal{X})=\frac{\lambda+2 \mu}{2 \mu^{2}},
$$

while we know from [11] that the relaxation time of the process is given by

$$
r(\mathcal{X})=\frac{\lambda+2 \mu+\sqrt{\lambda^{2}+4 \lambda \mu}}{2 \mu^{2}} .
$$

We will finally apply our results to the process of the number of customers in an $M / M / c$ queueing system - the setting in which Stadje and Parthasarathy [10] proposed the integral (1) as a measure of the speed of convergence to stationarity - and compare our findings with those in [10]. The process at hand is a birth-death process $\mathcal{X}$ with rates

$$
\lambda_{j}=\lambda \text { and } \mu_{j}=\min \{j, c\} \mu, \quad j \in \mathcal{N} .
$$

Writing

$$
\rho \equiv \frac{\lambda}{c \mu},
$$

we must have $\rho<1$ for the system to be stable. The potential coefficients of the process are given by

$$
\pi_{j}= \begin{cases}\frac{(c \rho)^{j}}{j !}, & 0 \leq j \leq c \\ \frac{c^{c} \rho^{j}}{c !}, & j \geq c\end{cases}
$$


so, with

$$
K_{c} \equiv \sum_{j=c}^{\infty} \pi_{j}=\frac{1}{1-\rho} \frac{(c \rho)^{c}}{c !}
$$

we have

$$
K=\sum_{j=0}^{c-1} \frac{(c \rho)^{j}}{j !}+K_{c} \text { and } E(X)=c \rho+\frac{\rho}{1-\rho} \frac{K_{c}}{K} .
$$

It is convenient to let

$$
A_{j} \equiv \sum_{k=0}^{j-1}\left(\lambda_{k} p_{k}\right)^{-1} \sum_{\ell=k+1}^{\infty} p_{\ell}, \quad j \geq 0
$$

(so that $A_{0} \equiv 0$ ), which is readily seen to imply

$$
A_{j}=\frac{1}{\lambda} \sum_{k=0}^{j-1} \frac{k !}{(c \rho)^{k}}\left(\sum_{\ell=k+1}^{c-1} \frac{(c \rho)^{\ell}}{\ell !}+K_{c}\right), \quad 0 \leq j \leq c .
$$

The quantities $\tau_{j}$ of (9) can now be expressed as

$$
\tau_{j}= \begin{cases}\frac{A_{j}}{K} \frac{(c \rho)^{j}}{j !}, & 0 \leq j \leq c, \\ \frac{1}{K}\left(A_{c}+(j-c) \frac{1}{\lambda} \frac{\rho}{1-\rho}\right) \frac{c^{c} \rho^{j}}{c !}, & j \geq c,\end{cases}
$$

from which it follows after some algebra that

$$
T=\frac{1}{K} \sum_{j=1}^{c-1} A_{j} \frac{(c \rho)^{j}}{j !}+T_{c}
$$

and

$$
\sum_{j=0}^{\infty} j \tau_{j}=\frac{c \rho}{K} \sum_{j=0}^{c-2} A_{j+1} \frac{(c \rho)^{j}}{j !}+T_{c}\left(c+\frac{\rho}{1-\rho}\right)+\frac{K_{c}}{K} \frac{1}{\lambda} \frac{\rho^{2}}{(1-\rho)^{3}},
$$

where

$$
T_{c} \equiv \sum_{j=c}^{\infty} \tau_{j}=\frac{K_{c}}{K}\left(A_{c}+\frac{1}{\lambda} \frac{\rho^{2}}{(1-\rho)^{2}}\right) .
$$

The integral (1) can now easily be evaluated for specific values of $c, \lambda$ and $\mu$ from (13) and the expressions (23) - (29). In particular, for $c=1$ we obtain

$$
\int_{0}^{\infty}[E(X)-E(X(t))] d t=\frac{1}{\mu} \frac{\rho}{(1-\rho)^{3}} .
$$

As a consequence the measure (3) for the $M / M / 1$ queue is given by

$$
m(\mathcal{X})=\frac{1}{\mu} \frac{1}{(1-\rho)^{2}}
$$


while it is well known that the relaxation time of the $M / M / 1$ queue satisfies

$$
r(\mathcal{X})=\frac{1}{\mu} \frac{(1+\sqrt{\rho})^{2}}{(1-\rho)^{2}} .
$$

Evaluating (12) for $c=2$ leads to

$$
\int_{0}^{\infty}[E(X)-E(X(t))] d t=\frac{1}{\mu} \frac{2 \rho\left(1-\rho+\rho^{2}\right)}{(1-\rho)^{3}(1+\rho)^{2}},
$$

so in this case we have

$$
m(\mathcal{X})=\frac{1}{\mu} \frac{1-\rho+\rho^{2}}{(1-\rho)^{2}(1+\rho)},
$$

while the relaxation time of the $M / M / 2$ queue is given in [1] as

$$
r(\mathcal{X})= \begin{cases}\frac{1}{\mu} \frac{2}{1+4 \rho+\sqrt{1-8 \rho}}, & 0<\rho<\frac{1}{9}, \\ \frac{1}{2 \mu} \frac{(1+\sqrt{\rho})^{2}}{(1-\rho)^{2}}, & \frac{1}{9} \leq \rho<1 .\end{cases}
$$

Comparing our results with those of Stadje and Parthasarathy [10], we find agreement for $c=1$, but a discrepancy for $c=2$. As a check, we evaluated the integral $I_{j}$ of (11) directly by using the representation for $p_{j}(t)$ derived in Karlin and McGregor [6] for $j=\lambda=\mu=1$, and found that it equals 0, which is consistent with (12), but not with Theorem 3 of Stadje and Parthasarathy [10].

\section{Discrete-time birth-death processes}

A discrete-time birth-death process or random walk $\tilde{\mathcal{X}} \equiv\{\tilde{X}(n), n=0,1, \ldots\}$ on the state space $\mathcal{N} \equiv\{0,1, \ldots\}$ is a Markov chain with stationary one-step transition probabilities $p_{i j}$ satisfying $p_{i j}=0$ for $|i-j|>1$. We shall only consider honest random walks in which $p_{j} \equiv p_{j, j+1}>0, q_{j+1} \equiv p_{j+1, j}>0$, and $r_{j} \equiv p_{j j} \geq 0$ for all $j \in \mathcal{N}$, but $r_{j}>0$ for at least one $j \in \mathcal{N}$ (the latter to avoid periodicity). We assume throughout that $\tilde{X}(0)=0$ and let

$$
\tilde{p}_{j}(n) \equiv \operatorname{Pr}(\tilde{X}(n)=j \mid \tilde{X}(0)=0), \quad j \in \mathcal{N}, n \geq 0 .
$$

Defining

$$
\tilde{\pi}_{0}=1 \text { and } \tilde{\pi}_{j}=\frac{p_{0} p_{1} \ldots p_{j-1}}{q_{1} q_{2} \ldots q_{j}}, \quad j \geq 1,
$$


it is well known that the process is ergodic if

$$
\tilde{K} \equiv \sum_{j=0}^{\infty} \tilde{\pi}_{j}<\infty,
$$

in which case

$$
\tilde{p}_{j} \equiv \lim _{n \rightarrow \infty} \tilde{p}_{j}(n)=\frac{\tilde{\pi}_{j}}{\tilde{K}}, \quad j \in \mathcal{N}
$$

and

$$
\lim _{n \rightarrow \infty} E(\tilde{X}(n))=E(\tilde{X})=\sum_{j=0}^{\infty} j \tilde{p}_{j}
$$

$\tilde{X}$ denoting a random variable with distribution $\left\{\tilde{p}_{j}, j \in \mathcal{N}\right\}$ (see, for example, Karlin and McGregor [7]).

If $E(\tilde{X})$ is finite it seems natural to propose - in analogy to (1) - the sum

$$
\sum_{n=0}^{\infty}[E(\tilde{X})-E(\tilde{X}(n))]
$$

as a measure of the speed of convergence of $\tilde{X}(n)$ to $\tilde{X}$, provided $E(\tilde{X}(n))$ converges monotonically to $E(\tilde{X})$. However, it is easy to construct examples of random walks starting in 0 in which the latter does not happen, so that (40) is less attractive than its continuous-time counterpart as a measure of the speed of convergence to stationarity. For completeness' sake we shall nevertheless evaluate the sum (40) explicitly, under the condition that

$$
E(\tilde{X}(n))<E(\tilde{X}), \quad n \geq 0 .
$$

We note that a sufficient condition for $E(\tilde{X}(n))$ to converge monotonically to its limit $E(\tilde{X})$ as $n \rightarrow \infty$ (and hence for (41)), is stochastic monotonicity of $\tilde{\mathcal{X}}$, which prevails if and only if

$$
p_{j}+q_{j+1} \leq 1, \quad j \in \mathcal{N}
$$

(see Kijima [9, Example 3.12]).

To evaluate the sum (40) we associate with $\tilde{\mathcal{X}}$ a continuous-time birth-death process $\mathcal{X} \equiv\{X(t), t \geq 0\}$ with rates

$$
\lambda_{j}=p_{j} \text { and } \mu_{j}=q_{j}, \quad j \in \mathcal{N} .
$$


Since $\lambda_{j}+\mu_{j}=p_{j}+q_{j} \leq 1$ for all $j$, the process $\mathcal{X}$ is uniformizable with uniformization parameter 1 and we get $\tilde{\mathcal{X}}$ back as the uniformized process. Moreover, with $\{N(t), t \geq 0\}$ denoting a Poisson process with intensity 1 , we have

$$
\{X(t), t \geq 0\} \stackrel{\mathrm{d}}{=}\{\tilde{X}(N(t)), t \geq 0\}
$$

(see, for example, [9, Section 4.4] for these results on uniformization). The next theorem shows that the problem of evaluating (40) can now be reduced to that of evaluating the integral (1) for the continuous-time process $\mathcal{X}$.

Theorem 2 If $E(\tilde{X}(n))<E(\tilde{X})$ for all $n \geq 0$, then

$$
\sum_{n=0}^{\infty}[E(\tilde{X})-E(\tilde{X}(n))]=\int_{0}^{\infty}[E(X)-E(X(t))] d t
$$

where $\{X(t), t \geq 0\}$ is the birth-death process with rates (43).

Proof. It is obvious from (44) that $E(\tilde{X})=E(X)$. Moreover, by conditioning on the value of $N(t)$ we get

$$
\begin{aligned}
& \int_{0}^{\infty}[E(X)-E(X(t))] d t=\int_{0}^{\infty}[E(\tilde{X})-E(\tilde{X}(N(t)))] d t \\
& =\int_{0}^{\infty}\left\{\sum_{n=0}^{\infty}[E(\tilde{X})-E(\tilde{X}(n))] e^{-t} \frac{t^{n}}{n !}\right\} d t=\sum_{n=0}^{\infty}[E(\tilde{X})-E(\tilde{X}(n))]
\end{aligned}
$$

where the interchange of integration and summation is allowed by Fubini's theorem.

Acknowledgement. This work was partly done while the second author was visiting the University of Durham.

\section{References}

[1] J.P.C. Blanc and E.A. van Doorn (1986). Relaxation times for queueing systems. In Mathematics and Computer Science, eds J.W. de Bakker, M. Hazewinkel and J.K. Lenstra. CWI Monographs 1, North-Holland, Amsterdam, pp. 139-162. 
[2] W. Feller (1967). An Introduction to Probability Theory and Its Applications, Vol. 1, 3rd edn. Wiley, New York.

[3] P.J. Holewijn and A. Hordijk (1975). On the convergence of moments in stationary Markov chains. Stoch. Processes Appl. 3, 55-64.

[4] S. Karlin and J.L. McGregor (1957). The differential equations of birthand-death processes, and the Stieltjes moment problem. Trans. Amer. Math. Soc. 85, 589-646.

[5] S. Karlin and J.L. McGregor (1957). The classification of birth and death processes. Trans. Amer. Math. Soc. 86, 366-400.

[6] S. Karlin and J.L. McGregor (1958). Many server queueing processes with Poisson input and exponential service times. Pacific J. Math. 8, 87-118.

[7] S. Karlin and J.L. McGregor (1959). Random walks. Illinois J. Math. 3, 66-81.

[8] J. Keilson (1971). Log-concavity and log-convexity in passage time densities of diffusion and birth-death processes. J. Appl. Probab. 8, 391-398.

[9] M. Kijima (1997). Markov Processes for Stochastic Modeling. Chapman \& Hall, London.

[10] W. Stadje and P.R. Parthasarathy (1999). On the convergence to stationarity of the many-server Poisson queue. J. Appl. Probab. 36, 546-557.

[11] E.A. van Doorn (1981). The transient state probabilities for a queueing model where potential customers are discouraged by queue length. J. Appl. Prob. 18, 499-506.

[12] E.A. van Doorn (1985). Conditions for exponential ergodicity and bounds for the decay parameter of a birth-death process. Adv. Appl. Probab. 17, 514-530. 
[13] E.A. van Doorn and P. Schrijner (1995). Geometric ergodicity and quasistationarity in discrete-time birth-death processes. J. Austral. Math. Soc. (B) $\mathbf{3 7}, 121-144$.

[14] W. Whitt (1992). Asymptotic formulas for Markov processes with applications to simulation. Operat. Res. 40, 279-291. 